\title{
DIAGNÓSTICO QUANTITATIVO E QUALITATIVO DA ARBORIZAÇÃO DAS PRAÇAS DE ARACAJU, SE ${ }^{1}$
}

Anderson Linhares de Souza², Robério Anastácio Ferreira², Anabel Aparecida de Mello², Débora da Rocha Plácido $^{3}$, Carla Zoaid Alves dos Santos² ${ }^{2}$ Dalva Angélica Santos da Graça², Pedro Paulo de Almeida Júnior $^{4}$, Soraia Stéfane Barbosa Barretto², Jolly Dayanne de Melo Dantas², José Welton Azevedo de Paula², Thiago Lima da Silva², Lucas Pedro Silva Gomes ${ }^{2}$

\begin{abstract}
RESUMO - Aracaju foi uma cidade planejada com simplicidade e rigor geométrico. Desde a sua fundação, ela vem passando por constantes alterações, havendo cada vez menos áreas verdes no ambiente urbano. A arborização urbana bem planejada é muito importante, pois as árvores trazem benefícios à cidade nos processos de ordens ecológica (clima e poluição), biológica (saúde física do homem) e psicológica (saúde mental do homem). Porém, a falta de planejamento dessa arborização e o uso inadequado de algumas espécies são empecilhos para se atingirem esses benefícios. Dessa forma, este trabalho foi realizado com o objetivo de avaliar a composição florística de 22 praças de Aracaju, SE, como forma de conhecer a atual situação de sua arborização e possibilitar intervenções para melhorar a adequação técnica do espaço verde urbano. O levantamento florístico foi realizado através de censo, no período de setembro de 2006 a julho de 2007, em cada praça, com o reconhecimento e identificação de todos os indivíduos arbóreos, utilizando-se as técnicas tradicionais de coleta e herborização. Realizou-se a identificação das espécies com o auxílio de literatura específica. Foram identificados 1.290 indivíduos, distribuídos em 20 famílias botânicas, 46 gêneros e 64 espécies, sendo nove espécies não identificadas. Das espécies identificadas, $58 \%$ são exóticas no território brasileiro e $42 \%$, espécies nativas. As praças apresentam similaridade entre si, com coeficiente de Jaccard variando de 0,00 a 0,66, e a Praça Almirante Tamandaré e a Praça Graccho Cardoso foram as de maior similaridade (66\%).
\end{abstract}

Palavras-chave: Arborização urbana, Similaridade florística e Planejamento urbano.

\section{QUANTITATIVE AND QUALITATIVE DIAGNOSTIC OF URBAN TREES SQUARES IN ARACAJU, SE, BRAZIL}

\begin{abstract}
Aracaju is a city that was planned with geometric precision and simplicity. Since its settlement, it has gone through constant changes in its urban environment. Due to these changes, fewer green areas remain in the urban environment. Well-planned urban afforestation is very important, once trees provide benefits to the city in ecological (climate and pollution), biological (physical health of human beings) and psychological (mental health of population) processes. However, the absence of afforestation planning and the use of inappropriate species are the main obstacles to achieving these benefits. This study was carried out to evaluate the floristic composition of twenty-two main squares in Aracaju-SE, as a way to know the current status and allow some interventions to improve the technical adequacy of urban green space. The floristic survey was conducted by means of a Census, from September 2006 until July 2007, in each square making the recognition and identification of all trees present using traditional methods and herborization.
\end{abstract}

\footnotetext{
${ }^{1}$ Recebido em 22.02.2010 e aceito para publicação em 31.10.2011.

${ }^{2}$ Universidade Federal de Sergipe, UFS, Brasil.E-mail: <agro.linhares@hotmail.com>, <raf@ufs.br>, <anabel_mello@yahoo.com.br>, <zoaid@hotmail.com>, <dalvasg@yahoo.com.br>,<stefanebarretto@bol.com.br>, <jollyday@hotmail.com>, <weltonze@hotmail.com>, <lima_tfn@yahoo.com.br>e <lucaspsgomes@hotmail.com>.

${ }^{3}$ Caixa Econômica Federal, Aracaju, SE, Brasil. E-mail: < deboraplacido@bol.com.br>.

${ }^{4}$ Emflors, Pará, Brasil. E-mail: <pedroalmeidajr@yahoo.com.br>.
} 
The identification of species was done with the help of literature. We identified 1,290 individuals belonging to 20 families, 46 genera and 64 species; 9 species could not be identified. 58\% of the identified species are exotic in the Brazilian territory and $42 \%$ are native species. The squares show similarity between them, with Jaccard coefficient ranging from 0.00 to 0.66, and the Almirante Tamandaré and Graccho Cardoso Squares had the greatest similarity (66\%).

Keywords: Urban trees, Floristic similarity and Urban planning.

\section{INTRODUÇÃO}

Ao longo da história brasileira, curta, mas com intensas transformações, a natureza foi vista pelos urbanizadores, tradicionais e orgânicos, de modo bastante diferenciado. De início, embora em pequena escala e com poucos impactos, a natureza foi destruída sistematicamente. Sob a égide do modo de produção capitalista, as cidades cresceram desmensuradamente e estrangularam as áreas verdes e os rios que entremeavam os seus bairros (CARVALHO, 2003).

As áreas não edificadas de uma cidade, de propriedade pública ou particular, independentemente de sua destinação e uso, são chamadas de espaços livres urbanos. As áreas verdes são um tipo especial de espaço livre urbano, onde os elementos fundamentais de composição são a vegetação e o solo livre de impermeabilização (GUZZO et al., 2006). Nesse sentido, as áreas destinadas à vegetação estão cada vez mais restritas aos canteiros centrais das avenidas, praças públicas e parques municipais.

Macedo e Robba (2003) afirmaram que as praças são espaços livres públicos urbanos destinados ao lazer e ao convívio da população, acessíveis aos cidadãos e livres de veículos. Nessas áreas, pode-se encontrar boa parcela dos indivíduos arbóreos nas cidades, porém é possível que não representem boa diversidade de espécies nem respeitem aos valores culturais, ambientais e de memória da cidade.

De acordo com Lira et al. (2004), o crescimento acelerado das cidades, resultante do acentuado aumento populacional, tem comprometido a quantidade e qualidade de seus espaços livres e áreas verdes. Assim, considerar a existência de benefícios econômicos e sociais das árvores nas cidades é apenas um processo lógico, uma vez que existem benefícios de ordens ecológica (clima e poluição), biológica (saúde física do homem) e psicológica (saúde mental do homem).

Com a alteração do ambiente original, as árvores poupadas no processo de urbanização e aquelas plantadas nesse novo cenário passam a lidar com condições ambientais adversas, muito diferentes das de seu ambiente original, diminuindo a expectativa de vida desses exemplares. A redução da biodiversidade, ocasionada pelo plantio de poucas espécies, também é fator preocupante, uma vez que torna a floresta urbana mais suscetível a doenças e pragas (SILVA et al., 2007a).

Deve-se considerar que, de acordo com a Sociedade Brasileira de Arborização Urbana, é necessário que as cidades apresentem $15 \mathrm{~m}^{2}$ de área verde por habitante, atendendo-se ao critério de proporcionar boa qualidade ambiental à população (SBAU, 2006).

O planejamento adequado para a arborização urbana, assim como a definição de metas quantitativas e qualitativas, é observado apenas em poucas cidades brasileiras (SILVA FILHO; BORTOLETO, 2005). Ainda, segundo esses autores, outro aspecto relevante está relacionado à baixa diversidade de espécies encontradas nas cidades brasileiras, prevalecendo a homogeneidade, que torna isso um grande risco de ocorrência de pragas e doenças, podendo ocasionar uma possível dizimação da população arbórea.

Gonçalves e Paiva (2004) afirmaram que, apesar da enorme variedade de espécies nativas de cada região, como é próprio do clima tropical, os planejadores têm preferido o uso de espécies exóticas, tanto de outras regiões do país quanto estrangeiras. Tal fato se deve, provavelmente, ao desconhecimento das espécies nativas e à comodidade de sugestão de espécies tidas como bem adaptadas em diversos lugares. Com isso, ocorre a uniformização das paisagens de diferentes cidades, reduzindo a biodiversidade no meio urbano e dissociando-o do contexto ambiental em que se insere (MACHADO et al., 2006).

As árvores trazem benefícios para a população que podem ser mensurados e avaliados, como: redução de ruído e da poluição atmosférica, melhoria no microclima e abrigo da fauna, além de colaborar com a estética da cidade (beleza paisagística) (SILVA FILHO et al., 2002; PAIVA; GONÇALVES, 2002; GONÇALVES

Revista Árvore, Viçosa-MG, v.35, n.6, p.1253-1263, 2011 
e PAIVA, 2004). O conhecimento da composição florística resultante da ação antrópica é imprescindível para dar suporte às ações que visam à preservação e melhoria da diversidade dessas áreas (SILVA et al., 2007a).

Conforme se pode observar nos estudos relacionados à identificação florística da arborização urbana de algumas cidades brasileiras, entre as espécies mais utilizadas destacam-se as da família Leguminosae, apresentando as três subfamílias abundantemente encontradas no Brasil: Caesalpinoideae, Mimosoideae e Papilionoideae (FARIA et al., 2007; SAMPAIO e DE ANGELIS 2008; SILVA et al., 2008; MOURA; SANTOS, 2009). Outra família muito utilizada, devido à floração intensa de algumas espécies, é a Bignoniaceae (CRHISTO; DIAS, 2007; FARIA et al., 2007). É importante também citar o uso das espécies da família Moraceae, principalmente Ficus benjamina e Ficus microcarpa (ROCHAetal., 2004; SILVAetal., 2002; CALIXTOJUNIOR et al., 2009), de origem exótica que apresentam rápido desenvolvimento (LORENZI et al., 2003), mas extremamente adaptadas às condições edafoclimáticas brasileiras.

Silva et al. (2007b) afirmaram que a instituição de padrões como espécies de portes alto, médio e baixo, muito comuns em manuais e guias de arborização, é insuficiente quando o trabalho envolve variáveis tão diferentes da malha urbana, juntamente com as peculiaridades ecológicas de cada espécie. Ainda, segundo esses autores, realizar inventários sobre a arborização das cidades é de fundamental importância no planejamento urbano, pois se devem considerar as características fitogeográficas, climáticas e pedológicas específicas de cada lugar.

O inventário da arborização urbana permite que os órgãos competentes tenham conhecimento da diversidade e do comportamento das espécies, bem como do controle de pragas e doenças, além do monitoramento de podas, plantios e manutenção em geral, fornecendo, assim, parâmetros de avaliação dos indivíduos arbóreos (SCHUCH, 2006).

Aracaju foi planejada especialmente para ser a sede do Governo Estadual, e desde a sua elevação à condição de capital, em 1855, a cidade foi marcada por grandes obras, como a construção de praças e a reforma das principais praças situadas no ponto de origem da nova capital (PORTO, 1945).
Com traçados e estilos arquitetônicos diferentes e sempre mantendo áreas ajardinadas e incorporando outros elementos paisagísticos, as praças mais antigas sofreram alterações significativas para acompanhar as necessidades e tendências de cada época. Mesmo assim, não houve a eliminação de indivíduos arbóreos e, sim, a incorporação de outros, exóticos ou nativos.

A diversidade da flora arbórea com indivíduos representativos da nossa região é um dos pontos importantes na caracterização cultural de nossas praças, uma vez que a introdução de espécies exóticas influenciou a arborização urbana da capital desde os seus primórdios, e a substituição dos indivíduos mortos ou doentes ao longo desse período não foi criteriosamente pensado.

Este trabalho foi realizado com o objetivo de contribuir para o maior conhecimento da composição florística da vegetação arbórea das principais praças da cidade de Aracaju, determinando sua similaridade florística.

\section{MATERIAL E MÉTODOS}

Este estudo foi realizado no município de Aracaju, SE, localizado nas coordenadas $10^{\circ} 55^{\prime} 00^{\prime \prime}$ de latitude Sul e $37^{\circ} 03^{\prime} 00^{\prime \prime}$ de latitude Oeste, a uma altitude de $4 \mathrm{~m}$ acima do nível do mar, com área territorial de $176 \mathrm{~km}^{2}$. O clima da região é do tipo A’s, segundo a classificação de Köppen, isto é, clima quente e chuvoso, com precipitação média anual normal em torno de $1.400 \mathrm{~mm}$ e temperatura máxima de $30^{\circ} \mathrm{C}$ e mínima de $23^{\circ} \mathrm{C}$, sendo a média ao redor de $26{ }^{\circ} \mathrm{C}$. O solo é do tipo Neossolo Quartzarênico (Arenoso), ácido e com baixa fertilidade natural (EMBRAPA, 1999). A vegetação original da região está inserida no domínio da Mata Atlântica (VELLOSO et al., 1991).

Foram selecionadas 22 praças do perímetro urbano da cidade como objeto deste estudo (Tabela 1 ).

O levantamento florístico foi realizado por meio de censo, no período de setembro de 2006 a julho de 2007, em que todos os indivíduos foram contados e identificados. O reconhecimento e identificação das espécies foram realizados com materiais existentes (exsicatas) no Laboratório de Dendrologia e Ecologia Florestal do Departamento de Ciências Florestais da Universidade Federal de Sergipe e por meio de consultas a literaturas especializadas (LORENZI et al., 1998, 2003, 2004).

Revista Árvore, Viçosa-MG, v.35, n.6, p.1253-1263, 2011 
Tabela 1 - Listagem das 22 principais praças de Aracaju, SE, cadastradas com os respectivos endereços e área. Table 1 - Listing of the twenty-two main squares in Aracaju-SE, registered with their addresses and area.

\begin{tabular}{|c|c|c|}
\hline Praça & Endereço & Área $\left(\mathrm{m}^{2}\right)$ \\
\hline Almirante Barroso & Bairro Centro & $12.052,70 \mathrm{~m}^{2}$ \\
\hline Almirante Tamandaré & Bairro São José & $749,45 \mathrm{~m}^{2}$ \\
\hline Assis Chateaubriand & Bairro Salgado Filho & $1.163,76 \mathrm{~m}^{2}$ \\
\hline Bandeira & Bairro Centro & $14.116,00 \mathrm{~m}^{2}$ \\
\hline Camerino & Bairro Centro & $10.731,45 \mathrm{~m}^{2}$ \\
\hline Dom José Tomaz & Bairro Siqueira Campos & $15.102,74 \mathrm{~m}^{2}$ \\
\hline Fausto Cardoso & Bairro Centro & $12.006,99 \mathrm{~m}^{2}$ \\
\hline General Valadão & Bairro Centro & $3.945,77 \mathrm{~m}^{2}$ \\
\hline Getúlio Vargas & Bairro São José & $6.607,00 \mathrm{~m}^{2}$ \\
\hline Graccho Cardoso & Bairro São José & $2.181,35 \mathrm{~m}^{2}$ \\
\hline Imprensa & Bairro 13 de Julho & $3.464,96 \mathrm{~m}^{2}$ \\
\hline Juscelino Kubitscheck & Bairro Jabotiana & $2.440,00 \mathrm{~m}^{2}$ \\
\hline Luciano Barreto Junior & Conjunto Jardins & $5.825,37 \mathrm{~m}^{2}$ \\
\hline Monteiro Lobato & Conjunto Inácio Barbosa & $10.237,70 \mathrm{~m}^{2}$ \\
\hline Olímpio Campos & Bairro Centro & $14.472,00 \mathrm{~m}^{2}$ \\
\hline Oliveira Belo & Bairro Gragerú & $6.070,70 \mathrm{~m}^{2}$ \\
\hline Poeta Clodoaldo de Alencar & Bairro Gragerú & $12.541,40 \mathrm{~m}^{2}$ \\
\hline Princesa Isabel & Bairro Santo Antônio & $5.822,90 \mathrm{~m}^{2}$ \\
\hline Siqueira Menezes & Bairro Santo Antônio & $1.918,58 \mathrm{~m}^{2}$ \\
\hline Teodorico Prado Montes & Farolândia & $4.513,50 \mathrm{~m}^{2}$ \\
\hline Tobias Barreto & Bairro São José & $14.431,10 \mathrm{~m}^{2}$ \\
\hline Rótula da Avenida Hermes Fontes & Bairro Ponto Novo & $5.389,00 \mathrm{~m}^{2}$ \\
\hline Total & & $165.784,42 \mathrm{~m}^{2}$ \\
\hline
\end{tabular}

As espécies exóticas foram definidas como aquelas que ocorrem numa área fora de seu limite natural historicamente conhecido, como resultado de dispersão acidental ou intencional por atividades antrópicas (ZILLER e DERBEDET, 2010). Para a classificação dessas áreas foram realizadas consultas à literatura especializada (LORENZI et al., 2003; GRAF, 2003; LORENZI et al., 2004; INSTITUTO HORUS, 2010).

Para a compilação, processamento e representação gráfica dos dados, empregou-se o software Microsoft Excel 2007.

A partir da presença (1) e ausência das espécies (0) em cada praça, foi construída uma matriz binária. Com essa matriz, calculou-se a estimativa de similaridade florística entre as praças, por meio do coeficiente de Jaccard, empregando o programa NTSYS pc2.1. (ROHLF, 2001). As similaridades obtidas foram calculadas empregando-se a seguinte expressão:

$$
S g_{i j}=\frac{a}{a+b+c}
$$

em que:

$a=$ número de espécies comuns nas praças i e j; $b=$ número espécies exclusivas na praça i; e

$c=$ número de espécies exclusivas da praça j.

A representação simplificada das similaridades foi realizada pela construção de um dendrograma pelo método de agrupamento Unweighted Pair-Group Method Arithmetic Average (UPGMA), utilizando o programa NTSYS pc2.1, versão freeware (ROHLF, 2001).

\section{RESULTADOS}

No levantamento florístico realizado nas 22 principais praças de Aracaju, SE, foram mensurados e identificados 1.290 indivíduos, os quais foram distribuídos em 20 famílias botânicas, 46 gêneros e 64 espécies, sendo nove espécies não identificadas por estarem em estágio vegetativo (Tabela 2).

Entre as famílias botânicas que mais contribuíram para a diversidade florística, pelo número de espécies, destacam-se: Leguminosae Caesalpinioideae, com nove espécies; Palmae (Arecaceae), com oito espécies; Leguminosae Mimosoideae, com cinco espécies; Bignoniaceae e Myrtaceae, com quatro espécies cada; Anacardiaceae, Leguminosae Papilionoideae e Moraceae, com três espécies cada; Annonaceae, Bombacaceae

Revista Árvore, Viçosa-MG, v.35, n.6, p.1253-1263, 2011 
Tabela 2 - Listagem florística das espécies arbóreas presentes nas principais praças de Aracaju, SE. Table 2 - Floristic list of tree species present in the main squares of Aracaju, SE.

\begin{tabular}{|c|c|c|}
\hline Família Botânica & Nome Científico & Nome Popular \\
\hline \multirow[t]{3}{*}{ Anacardiaceae } & Anacardium occidentale L. & Cajueiro \\
\hline & Mangifera indica L. & Mangueira \\
\hline & Schinus terebinthifolius Raddi. & Aroeira \\
\hline \multirow[t]{2}{*}{ Annonaceae } & Annona muricata L. & Graviola \\
\hline & Annona squamosa $\mathrm{L}$ & Pinha \\
\hline \multirow[t]{4}{*}{ Bignoniaceae } & Spathodea nilotica Seem & Espatódea \\
\hline & Tabebuia aurea (Silva Manso) Benth. \& Hook.f. ex Moore & Craibeira \\
\hline & Tabebuia impetiginosa (Mart. ex DC.) Standl. & Ipê-roxo \\
\hline & Tabebuia serratifolia (Vahl) G. Nichols & Ipê-amarelo \\
\hline Bombacaceae & Chorisia speciosa St. Hil. & Paineira \\
\hline (Malvaceae) & Pachira aquatica Aubl. & Castanha-do-maranhão \\
\hline Casuarinaceae & Casuarina equisetifolia J.R. \& G. Forst. & Casuarina \\
\hline Cecropiaceae (Urticaceae) & Cecropia pachystachya Trécul & Embaúba \\
\hline Combretaceae & Terminalia catappa $\mathrm{L}$. & Amendoeira \\
\hline Chrysobalanaceae & Licania tomentosa (Benth.) Fritsch & Oiti \\
\hline Leguminosae & Caesalpinia echinata Lam. & Pau-brasil \\
\hline \multirow[t]{8}{*}{ Caesalpinioideae } & Caesalpinia leiostachya (Benth.) Ducke & Pau-ferro \\
\hline & Caesalpinia peltophoroides Benth. & Sibipiruna \\
\hline & Cassia fistula $\mathrm{L}$. & Cássia-amarela \\
\hline & Cassia grandis L.f. & Canafístula \\
\hline & Senna siamea (Lam.) H.S. Irwin \& R.C. Barneby & Cássia seamea \\
\hline & Delonix regia (Bojer ex Hook.) Raf. & Flamboyant \\
\hline & Peltophorum dubium (Spreng.) Taub. & Angico-amarelo \\
\hline & Tamarindus indica $\mathrm{L}$. & Tamarindo \\
\hline Leguminosae & Adenanthera pavonina L. & Tento-carolina \\
\hline \multirow[t]{4}{*}{ Mimosoideae } & Anadenanthera colubrina (Vell.) Brenan & Angico-vermelho \\
\hline & Leucaena leucocephala (Lam.) R de Wit & Leucena \\
\hline & Pithecellobium dulce (Roxb.) Benth. & Mata-fome \\
\hline & Prosopis juliflora (Sw.)DC. & Algaroba \\
\hline Leguminosae & Andira fraxinifolia Benth. & Angelim \\
\hline \multirow[t]{2}{*}{ Papilionoideae } & Clitoria fairchildiana R.A. Howard. & Sombreiro \\
\hline & Erythrina indica Lam. & Brasileirinho \\
\hline \multirow[t]{2}{*}{ Meliaceae } & Azadirachta indica A. Juss. & Nim \\
\hline & Cedrela fissilis Vell. & Cedro \\
\hline \multirow[t]{3}{*}{ Moraceae } & Arthocarpus heterophyllus Lam. & Jaqueira \\
\hline & Ficus benjamina L. & Ficus \\
\hline & Ficus microcarpa L.f & Ficus \\
\hline Moringaceae & Moringa oleifera Lam. & Moringa \\
\hline \multirow[t]{4}{*}{ Myrtaceae } & Eucalyptus sp. & Eucalipto \\
\hline & Psidium guajava $\mathrm{L}$. & Goiabeira \\
\hline & Syzygium malaccense (L.) Merr. \& L.M. Perry & Jambo vermelho \\
\hline & Syzygium cumini (L.) Skeels & Jamelão \\
\hline \multirow[t]{9}{*}{ Palmae (Arecaceae) } & Cariota mitis Lour. & Palmeira rabo-de-peixe \\
\hline & Cocos nucifera L. & Coqueiro \\
\hline & Copernicia prunifera (Miller) H. E. Moore & Carnaúba \\
\hline & Dypsis lutescens H. Wendl & Areca-bambu \\
\hline & Elaeis guianeensis N. J. Jacquin. & Dendezeiro \\
\hline & Pritchardia pacifica Seemann e H. Wendl & Palmeira-leque de Fiji \\
\hline & Roystonea oleracea (N. J. Jacquin) O. F. Cook & Palmeira Real \\
\hline & Roystonea regia (H.B.K.) O.F. CooK & Palmeira Imperial \\
\hline & Syagrus coronata (Martius) Beccari & Dicuri \\
\hline
\end{tabular}


Tabela 2 - Cont.

Table 2 - Cont.

\begin{tabular}{lll} 
Rubiaceae & Genipa americana L. & Jenipapo \\
Rutaceae & Citrus aurantifolia Swingle & Limão \\
Sapindaceae & Sapindus saponaria L. & Saboneteira \\
Sterculiaceae (Malvaceae) & Sterculia chicha St. Hill. ex Turpin & Chichá \\
Não Identificadas & NI 1; Ni 2; NI 3; NI 4; NI 5; NI 6; NI 7; NI 8 e NI 9 & - \\
\hline
\end{tabular}

(Malvaceae) e Meliaceae, com duas espécies cada; e as outras nove famílias, que contribuíram com apenas uma espécie.

Os gêneros que apresentaram maior diversidade de espécies foram: Caesalpinia e Tabebuia, com três espécies cada; e Annona, Cassia, Ficus, Roystonea e Syzygium apresentaram duas espécies cada.

A maioria das espécies identificadas é exótica no território brasileiro, conforme definição de Ziller e Derbedet (2010), correspondendo a 58\%. As espécies nativas identificadas significaram $42 \%$ do total. Entre as espécies identificadas, 14 produzem frutos comestíveis, sendo Annona squamosa, Annona muricata, Arthocarpus heterophyllus, Citrus aurantifolia, Mangifera indica, Anacardium occidentale, Genipa americana e Psidium guajava cultivadas em plantios comerciais, pomares próximos à sede das propriedades rurais e quintais domésticos.

Considerando-se a quantidade de indivíduos distribuídos por espécie (Tabela 3), Pithecellobium dulce (205), Licania tomentosa (204), Clitoria fairchildiana (113) e Teminalia catappa (112) foram as mais representativas. Entretanto, as menos utilizadas foram Annona muricata, Arthocarpus heterophyllus, Chorisia speciosa, Copernicia prunifera, Erythrina indica, Psidium guajava e Tabebuia serratifolia, com apenas um indivíduo cadastrado cada uma.

Quanto à similaridade florística das praças, observou-se, por meio do coeficiente de Jaccard, uma variação de 0,00 a 0,66 (Figura 1), indicando maior semelhança entre as Praças Almirante Tamandaré e Graccho Cardoso (Tabela 4). Verificou-se a definição de um grupo formado pelas praças P1, P3, P6, P10, P13, P14, P16, P17 e P22, outro grupo constituído pelas praças P4, P5, P7, P12 e P19 e um terceiro pelas praças P2, P8, P15 e P21. A praça que apresentou o menor valor de similaridade comparada às outras foi a Assis Chateaubriand.

Na maioria das comparações entre as praças, o coeficiente de Similaridade de Jaccard obtido neste estudo foi significativo, pois, segundo Mueller-Dombois e Ellemberg (1974) citados por Ferreira et al. (2008), esse coeficiente raramente atinge valores acima de $60 \%$ e, quando superior a $25 \%$, duas amostras podem ser consideradas similares.

Tabela 3 - Número de indivíduos cadastrados por espécie nas principais praças de Aracaju, SE. Table 3 - Number of individuals registered by species in the main squares of Aracaju, SE.

\begin{tabular}{|c|c|c|c|}
\hline Nome Científico & Nome Popular & Quantidade & $(\%)$ \\
\hline Pithecellobium dulce & Mata-fome & 205 & $15,9 \%$ \\
\hline Licania tomentosa & Oiti & 204 & $15,8 \%$ \\
\hline Clitoria fairchildiana & Sombreiro & 113 & $8,8 \%$ \\
\hline Teminalia catappa & Amendoeira & 112 & $8,7 \%$ \\
\hline Roystonea regia & Palmeira imperial & 88 & $6,8 \%$ \\
\hline Ficus benjamina & Ficus & 82 & $6,4 \%$ \\
\hline Tamarindus indica & Tamarindo & 58 & $4,5 \%$ \\
\hline Ficus microcarpa & Ficus & 54 & $4,2 \%$ \\
\hline Mangifera indica & Mangueira & 44 & $3,4 \%$ \\
\hline Cocos nucifera & Coqueiro & 32 & $2,5 \%$ \\
\hline Syzygium cumini & Jamelão & 20 & $1,6 \%$ \\
\hline Schinus terebinthifolius & Aroeira & 17 & $1,3 \%$ \\
\hline
\end{tabular}

Revista Árvore, Viçosa-MG, v.35, n.6, p.1253-1263, 2011 
Tabela 3 - Cont.

Table 3 - Cont.

\begin{tabular}{|c|c|c|c|}
\hline Delonix regia & Flamboyant & 16 & $1,2 \%$ \\
\hline Prosopis juliflora & Algaroba & 16 & $1,2 \%$ \\
\hline Eucalyptus sp. & Eucalipto & 15 & $1,2 \%$ \\
\hline Andira fraxinifolia & Angelim & 12 & $0,9 \%$ \\
\hline Caesalpinia echinata & Pau-Brasil & 12 & $0,9 \%$ \\
\hline Cassia fistula & Cássia-amarela & 12 & $0,9 \%$ \\
\hline Casuarina equisetifolia & Casuarina & 12 & $0,9 \%$ \\
\hline Elaeis guianeensis & Dendezeiro & 12 & $0,9 \%$ \\
\hline Azadirachta indica & Nim & 11 & $0,9 \%$ \\
\hline Pritchardia pacifica & Palmeira-leque de Fiji & 11 & $0,9 \%$ \\
\hline Adenanthera pavonina & Tento carolina & 9 & $0,7 \%$ \\
\hline Anacardium occidentale & Cajueiro & 9 & $0,7 \%$ \\
\hline Pachira aquática & Castanha-do-maranhão & 9 & $0,7 \%$ \\
\hline Spathodea nilotica & Espatodea & 9 & $0,7 \%$ \\
\hline Dypsis lutescens & Areca bambu & 8 & $0,6 \%$ \\
\hline Tabebuia áurea & Craibeira & 8 & $0,6 \%$ \\
\hline Anadenanthera colubrina & Angico-vermelho & 7 & $0,5 \%$ \\
\hline Caesalpinia leiostachya & Pau-ferro & 7 & $0,5 \%$ \\
\hline Annona squamosa & Pinha & 5 & $0,4 \%$ \\
\hline Caesalpinia peltophoroides & Sibipiruna & 4 & $0,3 \%$ \\
\hline Cassia grandis & Canafístula & 4 & $0,3 \%$ \\
\hline Peltophorum dubium & Angico-amarelo & 4 & $0,3 \%$ \\
\hline Sapindus saponaria & Saboneteira & 4 & $0,3 \%$ \\
\hline Syzygium malaccense & Jambo vermelho & 4 & $0,3 \%$ \\
\hline Tabebuia impetiginosa & Ipê-roxo & 4 & $0,3 \%$ \\
\hline Cariota mitis & Palmeira rabo de peixe & 3 & $0,2 \%$ \\
\hline Senna siamea & Cassia siâmea & 3 & $0,2 \%$ \\
\hline Genipa americana & Jenipapeiro & 3 & $0,2 \%$ \\
\hline Moringa oleifera Lam. & Moringa & 3 & $0,2 \%$ \\
\hline Sterculia chicha & Chichá & 3 & $0,2 \%$ \\
\hline Cecropia pachystachya & Embaúba & 2 & $0,2 \%$ \\
\hline Cedrela fissilis & Cedro & 2 & $0,2 \%$ \\
\hline Citrus aurantifolia & Limoeiro & 2 & $0,2 \%$ \\
\hline Citrus sinensis & Laranja & 2 & $0,2 \%$ \\
\hline Leucaena leucocephala & Leucena & 2 & $0,2 \%$ \\
\hline Syagrus coronata & Dicuri & 2 & $0,2 \%$ \\
\hline Annona muricata & Graviola & 1 & $0,1 \%$ \\
\hline Arthocarpus heterophyllus & Jaqueira & 1 & $0,1 \%$ \\
\hline Chorisia speciosa & Paineira & 1 & $0,1 \%$ \\
\hline Copernicia prunifera & Carnaúba & 1 & $0,1 \%$ \\
\hline Erythrina indica & Brasileirinho & 1 & $0,1 \%$ \\
\hline Psidium guajava & Goiabeira & 1 & $0,1 \%$ \\
\hline Tabebuia serratifolia & Ipê amarelo & 1 & $0,1 \%$ \\
\hline Total & & 1290 & $100,0 \%$ \\
\hline
\end{tabular}

\section{DISCUSSÃO}

A realização de inventários sobre a arborização urbana é fundamental para um bom planejamento nesse ambiente, pois para o uso adequado das árvores urbanas devem-se considerar tanto as características fitogeográficas de cada região quanto as climáticas e pedológicas específicas de cada lugar (SILVA et al., 2007b). Nesse sentido, o conhecimento das espécies que foram utilizadas nas principais praças da cidade de Aracaju, SE, pode contribuir para a realização de projetos futuros de implantação de novas praças ou, ainda, para promover possíveis adequações, em caso de necessidade de substituição de indivíduos. 


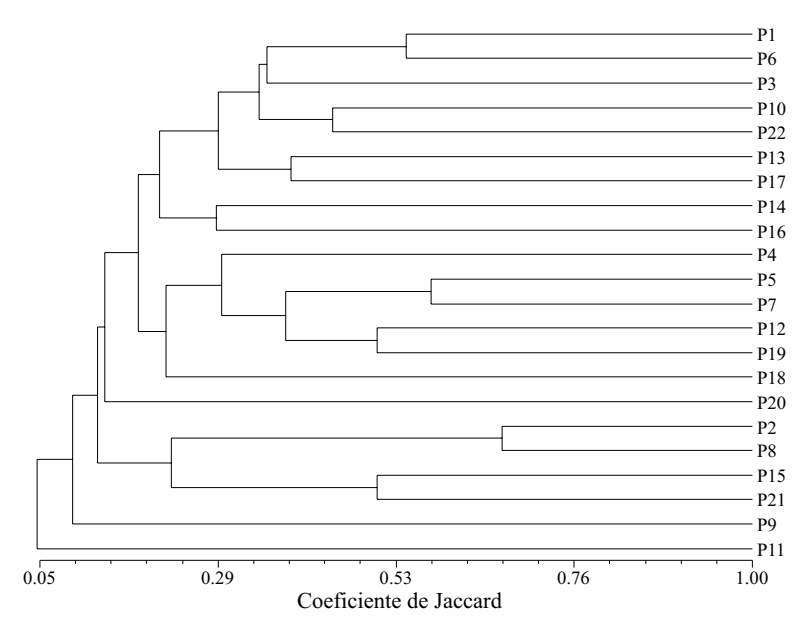

Figura 1 - Similaridade entre 22 principais praças localizadas na cidade de Aracaju, SE, definida pelo critério de agrupamento UPGMA, com base na média do índice de Jaccard: P1 - Praça Almirante Barroso; P2 - Praça Almirante Tamandaré; P3 - Praça Monteiro Lobato; P4 - Praça da Bandeira; P5 - Praça da Imprensa; P6 - Praça Fausto Cardoso; P7 - Praça Getúlio Vargas; P8 - Praça Graccho Cardoso; P9 - Praça Oliveira Belo; P10 - Praça Camerino; P11 - Praça Assis Chateaubriand; P12 - Praça Teodorico Prado Montes; P13 - Praça Tobias Barreto; P14 - Praça Dom José Tomaz; P15 - Praça Siqueira Menezes; P16 - Praça Juscelino Kubitscheck; P17 - Praça Olímpio Campos; P18 - Praça da Rotula da Hermes Fontes; P19 - Praça Poeta Clodoaldo de Alencar; P20 - Praça Luciano Barreto Junior; P21 - Praça General Valadão; e P22 - Praça Princesa Isabel.

Figure 1 - Similarity between 22 main squares located in the city of Aracaju, SE, defined by the UPGMA clustering criterion, based on the average of the Jaccard index. P1 - Praça Almirante Barroso; P2 - Praça Almirante Tamandaré; P3 - Praça Monteiro Lobato; P4 - Praça da Bandeira; P5 - Praça da Imprensa; P6 - Praça Fausto Cardoso; P7 - Praça Getúlio Vargas; P8 - Praça Graccho Cardoso; P9 - Praça Oliveira Belo; P10 - Praça Camerino; P11 - Praça Assis Chateaubriand; P12 - Praça Teodorico Prado Montes; P13 - Praça Tobias Barreto; P14 - Praça Dom José Tomaz; P15-Praça Siqueira Menezes; P16-Praça Juscelino Kubitscheck; P17 - Praça Olímpio Campos; P18 - Praça da Rotula da Hermes Fontes; P19 - Praça Poeta Clodoaldo de Alencar; P20 - Praça Luciano Barreto Junior; P21 - Praça General Valadão; and P22 - Praça Princesa Isabel.

As informações obtidas são relevantes, pois no passado algumas espécies foram plantadas nas praças mais por sua disponibilidade de mudas na época de implantação do que por critérios técnicos de adequação ao espaço urbano. Muitas vezes, não se observou a relação das espécies escolhidas com aspectos como rede elétrica, largura dos passeios e aspectos como podas, sem considerar a altura adequada para o trânsito de pessoas e reduzindo o sombreamento desses espaços.

Pode-se observar que o número de espécies encontradas nas principais praças de Aracaju foi superior ao observado por Rossatto et al. (2008), em inventário realizado na cidade de Assis, SP. Esses autores encontraram 1.915 indivíduos arbóreos, pertencentes a 54 espécies, sendo as 10 mais frequentes responsáveis por $82,77 \%$ das plantas levantadas. A espécie mais frequente foi Licania tomentosa, com 21,41\% dos indivíduos. Considerando-se o número de espécies, as exóticas foram predominantes na arborização da cidade com 31 espécies, representando 61,82\%. Apesar de se tratar de cidades em regiões e climas diferentes, observa-se, na maioria dos trabalhos realizados com arborização urbana, um grupo de famílias botânicas e espécies comumente empregadas com essa finalidade.

À semelhança de Assis, SP, em Aracaju a Licania tomentosa também é uma espécie bastante utilizada e representa 15,8\% de todos os indivíduos inventariados, sendo inferior apenas a Pithecelobium dulce (15,9\%), que foi a espécie de maior ocorrência registrada. Deve-se considerar ainda que a presença de espécies exóticas em Aracaju (58\%) também foi superior às nativas (42\%), como observado por Rossato et al. (2008) na cidade de Assis, SP.

Em trabalho realizado por meio de censo no município de Lavras da Mangabeira, no Estado do Ceará, Calixto Júnior et al. (2009) verificaram a presença de 2.784 indivíduos arbóreos, estando distribuídos em 22 espécies, 21 gêneros e 9 famílias botânicas. Entre as espécies identificadas, esses autores observaram que Ficus benjamina (42,42\%), Senna siamea (26,90\%) e Azadiractha indica (23,63\%) eram predominantes e totalizavam 92,95\% dos indivíduos inventariados. Entre as famílias botânicas encontradas, a mais representativa e com maior número de espécies foi a Fabaceae (11), com as subfamílias Caesalpinioideae (7), Mimosoideae (3) e Papilionoideae (1). No entanto, a família que apresentou o maior número de indivíduos foi Moraceae (1.181). 
Tabela 4 - Matriz de similaridade entre as 22 principais praças localizadas na Cidade de Aracaju, SE: P1 - Praça Almirante Barroso; P2 - Praça Almirante Tamandaré; P3 - Praça Monteiro Lobato; P4 - Praça da Bandeira; P5 - Praça da Imprensa; P6 - Praça Fausto Cardoso; P7 - Praça Getúlio Vargas; P8 - Praça Graccho Cardoso; P9 - Praça Oliveira Belo; P10 - Praça Camerino; P11 - Praça Assis Chateaubriand; P12 - Praça Teodorico Prado Montes; P13 - Praça Tobias Barreto; P14 - Praça Dom José Tomaz; P15 - Praça Siqueira Menezes; P16 - Praça Juscelino Kubitscheck; P17 - Praça Olímpio Campos; P18 - Praça da Rotula da Hermes Fontes; P19 - Praça Poeta Clodoaldo de Alencar; P20 - Praça Luciano Barreto Junior; P21 - Praça General Valadão; e P22 - Praça Princesa Isabel.

Table 4 - Matrix of similarity between the 22 main squares located in the city of Aracaju, SE. P1 - Praça Almirante Barroso; P2 - Praça Almirante Tamandaré; P3 - Praça Monteiro Lobato; P4 - Praça da Bandeira; P5 - Praça da Imprensa; P6 - Praça Fausto Cardoso; P7 - Praça Getúlio Vargas; P8 - Praça Graccho Cardoso; P9 - Praça Oliveira Belo; P10 - Praça Camerino; P11 - Praça Assis Chateaubriand; P12 - Praça Teodorico Prado Montes; P13 - Praça Tobias Barreto; P14 - Praça Dom José Tomaz; P15 - Praça Siqueira Menezes; P16 - Praça Juscelino Kubitscheck; P17 - Praça Olímpio Campos; P18 - Praça da Rotula da Hermes Fontes; P19 - Praça Poeta Clodoaldo De Alencar; P20 - Praça Luciano Barreto Junior; P21 - Praça General Valadão; and P22 - Praça Princesa Isabel.

\begin{tabular}{|c|c|c|c|c|c|c|c|c|c|c|c|c|c|c|c|c|c|c|c|c|c|c|}
\hline & P22 & P21 & P20 & P19 & P18 & P17 & P16 & P15 & P14 & P13 & P12 & P11 & P10 & P9 & P8 & P7 & P6 & P5 & $\mathrm{P} 4$ & P3 & $\mathrm{P} 2$ & P1 \\
\hline$\overline{\mathrm{P} 1}$ & & & & & & & & & & & & & & & & & & & & & & 1,00 \\
\hline P2 & & & & & & & & & & & & & & & & & & & & & 1,00 & 0,20 \\
\hline P3 & & & & & & & & & & & & & & & & & & & & 1,00 & 0,07 & 0,35 \\
\hline P4 & & & & & & & & & & & & & & & & & & & 1,00 & 0,21 & 0,20 & 0,11 \\
\hline P5 & & & & & & & & & & & & & & & & & & 1,00 & 0,23 & 0,26 & 0,14 & 0,14 \\
\hline P6 & & & & & & & & & & & & & & & & & 1,00 & 0,14 & 0,25 & 0,35 & 0,20 & 0,53 \\
\hline P7 & & & & & & & & & & & & & & & & 1,00 & 0,15 & 0,57 & 0,25 & 0,20 & 0,16 & 0,15 \\
\hline P8 & & & & & & & & & & & & & & & 1,00 & 0,14 & 0,18 & 0,28 & 0,30 & 0,14 & 0,66 & 0,18 \\
\hline P9 & & & & & & & & & & & & & & 1,00 & 0,12 & 0,10 & 0,00 & 0,20 & 0,14 & 0,26 & 0,00 & 0,00 \\
\hline $\mathrm{P} 10$ & & & & & & & & & & & & & 1,00 & 0,07 & 0,13 & 0,16 & 0,37 & 0,20 & 0,22 & 0,28 & 0,08 & 0,32 \\
\hline P11 & & & & & & & & & & & & 1,00 & 0,04 & 0,00 & 0,00 & 0,00 & 0,09 & 0,00 & 0,09 & 0,07 & 0,00 & 0,00 \\
\hline P12 & & & & & & & & & & & 1,00 & 0,14 & 0,20 & 0,20 & 0,28 & 0,37 & 0,14 & 0,50 & 0,33 & 0,26 & 0,14 & 0,06 \\
\hline P13 & & & & & & & & & & 1,00 & 0,16 & 0,08 & 0,35 & 0,03 & 0,08 & 0,16 & 0,32 & 0,11 & 0,17 & 0,33 & 0,08 & 0,32 \\
\hline P14 & & & & & & & & & 1,0 & 0,27 & 0,33 & 0,06 & 0,27 & 0,11 & 0,21 & 0,18 & 0,26 & 0,25 & 0,20 & 0,28 & 0,14 & 0,20 \\
\hline P15 & & & & & & & & 1,00 & 0,2 & & 0,00 & 0,00 & 0,13 & 0,00 & 0,20 & 0,14 & 0,30 & 0,12 & 0,08 & 0,14 & 0,25 & 0,30 \\
\hline P16 & & & & & & & 1,00 & 0,00 & 0,28 & 0,12 & 0,26 & 0,07 & 0,12 & 0,05 & 0,14 & 0,05 & 0,15 & 0,11 & 0,15 & 0,23 & 0,07 & 0,15 \\
\hline P17 & & & & & & 1,00 & 0,18 & 0,06 & 0,28 & 0,38 & 0,15 & 0,03 & 0,28 & 0,02 & 0,03 & 0,12 & 0,24 & 0,15 & 0,07 & 0,22 & 0,03 & 0,28 \\
\hline P18 & & & & & 1,00 & 0,18 & 0,16 & 0,00 & 0,22 & 0,24 & 0,40 & 0,11 & 0,10 & 0,07 & 0,10 & 0,18 & 0,12 & 0,16 & 0,12 & 0,16 & 0,11 & 0,06 \\
\hline P19 & & & & 1,00 & 0,21 & 0,17 & 0,29 & 0,00 & 0,21 & 0,14 & 0,50 & 0,10 & 0,23 & 0,25 & 0,20 & 0,27 & 0,18 & 0,36 & 0,35 & 0,37 & 0,10 & 0,18 \\
\hline P20 & & & 1,00 & 0,09 & 0,09 & 0,09 & 0,03 & 0,12 & 0,16 & 0,18 & 0,10 & 0,00 & 0,26 & 0,10 & 0,12 & 0,05 & 0,19 & 0,10 & 0,19 & 0,16 & 0,06 & 0,13 \\
\hline P21 & & 1,00 & 0,12 & 0,00 & 0,00 & 0,06 & 0,00 & 0,50 & 0,13 & 0,13 & 0,00 & 0,00 & 0,08 & 0,00 & 0,20 & 0,00 & 0,30 & 0,00 & 0,08 & 0,14 & 0,25 & 0,30 \\
\hline P22 & 1,00 & 0,23 & 0,16 & 0,22 & 0,16 & 0,18 & 0,08 & 0,14 & 0,28 & 0,33 & 0,18 & 0,07 & 0,44 & 0,11 & 0,14 & 0,12 & 0,35 & 0,10 & 0,27 & 0,36 & 0,15 & 0,35 \\
\hline
\end{tabular}

Em Aracaju, as famílias botânicas mais representativas em diversidade florística pelo número de espécies foram Leguminosae Caesalpinioideae (9 espécies), Palmae (Arecaceae -8 espécies), Leguminosae Mimosoideae (5 espécies), Bignoniaceae e Myrtaceae (4 espécies cada) e Anacardiaceae, Leguminosae Papilionoideae e Moraceae (3 espécies cada). As Leguminosae (Fabaceae), semelhantes às de Lavras da Mangabeira, CE, também eram predominantes em Aracaju.

Diferentemente de Aracaju, SE, de acordo com Silva et al. (2007c), em trabalho realizado em sete parques urbanos da cidade de Recife, PE, foram reconhecidas 87 espécies de árvores, arvoretas e palmeiras, obtendo-se 2.808 indivíduos, número de espécies superior ao observado em Aracaju. Segundo esses autores, foram registrados 2.087 exemplares de árvores e arvoretas nos sete parques, e as espécies mais abundantes foram sombreiro (Clitoria fairchildiana), mangueira (Mangifera indica), pau-d'arquinho (Tecoma stans), castanhola (Terminalia catappa) e ipê-roxo (Tabebuia impetiginosa), que reuniram $26,5 \%$ de todos os indivíduos. No entanto, a família Leguminosae também foi predominante em Recife, assim como em Aracaju. Na capital pernambucana, as espécies foram agrupadas em 35 famílias botânicas, e as famílias de maior riqueza foram Caesalpiniaceae, com 11 espécies, e Mimosaceae, com 10 espécies, seguidas de Myrtaceae (7 espécies) e Bignoniaceae e Moraceae, com cinco espécies cada (SILVA et al., 2007c).

Quanto aos aspectos da baixa diversidade de espécies, observada nas Praças de Aracaju, infere-se que, se esse fator não for considerado no planejamento urbano e na adequação da arborização, ele pode

Revista Árvore, Viçosa-MG, v.35, n.6, p.1253-1263, 2011 
comprometer esses espaços no futuro pela falência fisiológica das espécies num mesmo período, em relação ao seu ciclo de vida. Nesse caso, seria necessário promover a substituição de grande número de indivíduos numa mesma época, o que não é uma condição ideal nos espaços urbanos.

A alta similaridade florística observada nas praças de Aracaju se deve ao plantio de um grupo reduzido de espécies. Desse modo, se não houver planejamento adequado para a substituição gradativa dessas árvores, muitas das praças avaliadas poderão ficar desprovidas de sombreamento temporariamente, o que acarretará possíveis elevações da temperatura local, criando novas ilhas de calor.

\section{CONCLUSÕES}

As espécies Pithecellobium Dulce, Licania tomentosa, Clitoria fairchildiana e Teminalia catappa foram as mais representativas na arborização urbana das principais praças de Aracaju, SE. Entretanto, as menos utilizadas foram Annona muricata, Arthocarpus heterophyllus, Chorisia speciosa, Copernicia prunifera, Erythrina indica, Psidium guajava e Tabebuia serratifolia. Já as famílias mais representativas foram Leguminosae Casesalpinioideae, Palmae (Arecaceae), Leguminosae Mimosoideae, Bignoniaceae e Myrtaceae.

A maioria das espécies observadas é exótica no território brasileiro, correspondendo a 58\% daquelas identificadas. As espécies nativas identificadas correspondem a $42 \%$ do total.

As praças da capital sergipanas apresentaram similaridade entre si, sendo as Praças Almirante Tamandaré e Graccho Cardoso as de maior similaridade.

\section{REFERÊNCIAS}

CALIXTO JÚNIOR, J. A.; SANTANA, G. M.; LIRA FILHO, J. A. Análise quantitativa da arborização urbana de Lavras da Mangabeira, CE, Nordeste do Brasil. Revista da Sociedade Brasileira de Arborização Urbana, v.4, n.3, p.99-109, 2009. (Nota Técnica).

CARVALHO, P. F. Repensando as áreas verdes urbanas. Rio Claro: Unesp, Território \& Cidadania, 2003.
CRHISTO, J. A.; DIAS, A. N. Inventário florestal da arborização urbana do centro da cidade de Prudentópolis - PR. R. Eletrônica Lato Sensu, v.2, n.1, p.76-93, 2007. Disponível em: http://www.unicentro.br - Engenharia. Acesso em: 15 de ago. de 2010.

EMPRESA BRASILEIRA DE PESQUISA AGROPECUÁRIA - EMBRAPA. Sistema brasileiro de classificação de solos. Rio de Janeiro: 1999. 412p.

FARIA, J. L. G.; MONTEIRO, E. A.; FISCH, S. T. V. Arborização de vias públicas de município de Jacareí - SP. Revista da Sociedade Brasileira de Arborização Urbana, v.2, n.4, p.20-33, 2007.

FERREIRA, R. L. C. et al. Comparação de duas metodologias multivariadas no estudo de similaridade entre fragmentos de Floresta Atlântica. Revista Árvore, v.32, n.3, p.511521, 2008.

GONÇALVES, W.; PAIVA, H. N. Árvores para ambiente urbano. Viçosa, MG: Aprenda Fácil, 2004. v.2. 242p. (Coleção Jardinagem e Paisagismo).

GRAF, A. B. Tropica: Color Cyclopedia of Exotic plants and trees. 5.ed. Farmingdale: ROEHRS, 2003. 1152p.

GUZZO, P.; CARNEIRO, R. M. A.; OLIVEIRA JÚNIOR, H. O. Cadastro municipal de espaços livres urbanos de Ribeirão Preto (SP): acesso público, índices e base para novos instrumentos e mecanismos de gestão. Revista da

Sociedade Brasileira de Arborização Urbana, v.1, n.1, p.19-30, 2006.

INSTITUTO HORUS. Link de consulta de espécies exóticas e invasoras. Disponivel em: http://i3n.institutohorus.org.br/

filt_especies.asp. Acesso em 29/05/2010.

LORENZI, H. et al. Árvores Brasileiras: manual de identificação e cultivo de plantas arbóreas nativas do Brasil. 4.ed. Nova Odessa: Plantarum, 1998. v.1-2.

LORENZI, H. et al. Árvores exóticas no Brasil. Nova Odessa: Plantarum, 2003. 242p. 
LORENZI, H.et al. Palmeiras brasileiras e exóticas cultivadas. Nova Odessa: Plantarum, 2004.

LIRA, R.S. et al. Diagnóstico paisagístico do Parque da Criança em Campina Grande, PB. Revista de Biologia e Ciências da Terra, v.4. n.1, p.1-23, 2004.

MACEDO, S.S.; ROBBA, F. Praças brasileiras. Public Squares in Brazil. São Paulo: Edusp, 2003. 311p.

MACHADO, R. R. B. et al. Árvores nativas para a arborização de Teresina - Piauí. Revista da Sociedade Brasileira de Arborização Urbana, v.1, n.1, p.10-18, 2006.

MOURA, T.A.; SANTOS, V.L.L.V. Levantamento quali-quantitativo de espécies arbóreas e arbustivas na arborização viária urbana dos bairros centro e centro norte, Várzea Grande, Mato Grosso, Brasil. Revista da Sociedade Brasileira de Arborização Urbana, v.1, n.1, p.97-117, 2009.

PAIVA, H. N.; GONÇALVES, W. Florestas urbanas: planejamento para melhoria da qualidade de vida. Viçosa, MG: Aprenda Fácil, 2002. 180p.

PORTO, F. F. A cidade do Aracaju (1855/ 1865): ensaio de evolução urbana. Aracaju: Regina, 1945. v.2. 62p. (Estudos Sergipanos).

ROCHA, R. T.; LELES, P. S. S.; OLIVEIRA NETO, S. N. Arborização de vias públicas em Nova Iguaçu, RJ: o caso dos bairros Rancho Novo e Centro. Revista Árvore, v.28, n.4, p.599-607, 2004.

ROHLF, F. J. Numerical taxonomy and multivariate analysis system. Version 2.1. New York: Exeter Software, 2001. 38p.

ROSSATTO, D. R.; TSUBOY, M. S. F.; FREI, F. Arborização urbana na cidade de Assis-SP: uma abordagem quantitativa. Revista da Sociedade Brasileira de Arborização Urbana, v.3, n.3, p.1-16, 2008.

SAMPAIO, A. C. F.; DE ANGELIS, B. L. Inventário e análise da arborização de vias públicas de Maringá-PR. Revista da Sociedade Brasileira de Arborização urbana, v.3, n.1, p.37-57, 2008.
SCHUCH, M. I. S. Arborização urbana: uma contribuição à qualidade de vida com uso de geotecnologias. Santa Maria, Universidade Federal de Santa Maria, 2006. p.17-37.

SILVA FILHO, D. F.; BORTOLETO, S. Uso de indicadores de diversidade na definição de plano de manejo da arborização viária de Águas de São Pedro - SP. Revista Árvore, v.29, n.6, p.973982, 2005.

SILVA FILHO, D. F. et al. Banco Relacional para Cadastro, Avaliação e Manejo da Arborização em Vias Públicas. Revista Árvore, v.26, n.5, p.629-612, 2002

SILVA, A. G.; PAIVA, H. N.; GONÇALVES, W. Avaliando a arborização urbana. Viçosa, MG: Aprenda Fácil, 2007a. 346p.

SILVA, L. M. et al. Arborização de vias públicas e a utilização de espécies exóticas: o caso do Bairro Centro de Pato Branco/PR. Scientia Agraria, v.8, n.1, p.47-53, 2007b.

SILVA, L. R.; MEUNIER, I. M. J.; FREITAS, A. M. M. Riqueza e densidade de árvores, arvoretas e palmeiras em parques urbanos de Recife, Pernambuco, Brasil. Revista da Sociedade Brasileira de Arborização Urbana, v.2, n.4, p.34-49, 2007c.

SILVA, M. D. M.; SILVEIRA, R. P.; TEIXEIRA, M. I. J. G. Avaliação da arborização de vias públicas de uma área da região oeste da cidade de Franca/ SP. Revista da Sociedade Brasileira de Arborização Urbana, v.3, n.1, p.19-35, 2008.

SOCIEDADE BRASILEIRA DE ARBORIZAÇÃO URBANA - SBAU. "Carta a Londrina e Ibiporã". Boletim Informativo, v.3, n.5, p.3, 1996.

VELOSO, H. P.; RANGEL-FILHO, A. L. R.; LIMA, J. C. A. Classificação da vegetação brasileira adaptada a um sistema universal. Rio de Janeiro: IBGE, 1991. 123p.

ZILLER, S. R.; DEBERDT, A. J. Espécies Exóticas invasoras em unidades de conservação. Disponível em <http:// www.icmbio.gov.br/ChicoMendes/Artigos/ Artigo_EEI_em_UCs.pdf $>$ Acesso em 29 de maio de 2010 .

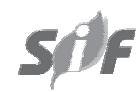

Revista Árvore, Viçosa-MG, v.35, n.6, p.1253-1263, 2011 
